\title{
Oscillatory Instabilities of Standing Waves in One-Dimensional Nonlinear Lattices
}

\author{
Anna Maria Morgante, Magnus Johansson, Georgios Kopidakis, and Serge Aubry \\ Laboratoire Léon Brillouin (CEA-CNRS), CEA Saclay, F-91191 Gif-sur-Yvette Cedex, France
}

(April 18, 2000)

\begin{abstract}
In one-dimensional anharmonic lattices, we construct nonlinear standing waves (SWs) reducing to harmonic SWs at small amplitude. For SWs with spatial periodicity incommensurate with the lattice period, a transition by breaking of analyticity versus wave amplitude is observed. As a consequence of the discreteness, oscillatory linear instabilities, persisting for arbitrarily small amplitude in infinite lattices, appear for all wave numbers $Q \neq 0, \pi$. Incommensurate analytic SWs with $|Q|>\pi / 2$ may however appear as 'quasi-stable', as their instability growth rate is of higher order.
\end{abstract}

PACS numbers: 63.20.Ry, 45.05.+x, 05.45.-a, 42.65.Sf

A wellknown and rather spectacular phenomenon occuring in many nonlinear media (e.g. fluids or optical waveguides) is the modulational (Benjamin-Feir) instability (MI), by which a travelling plane wave breaks up into a train of solitary waves (see e.g. [1]). It is also wellknown that wave propagation in many continuous nonlinear media is well described by Nonlinear Schrödinger type equations, where the solitary wave trains are described by spatially periodic and stable standing wave (SW) solutions, the so-called cnoidal envelope waves [2].

An analogous instability of propagating waves occurs also in discrete systems (e.g. anharmonic lattices), where in the case of soft (hard) anharmonicity, waves with small (large) wave numbers are unstable [3,4] and typically break up into arrays of intrinsically localized modes or discrete breathers (see e.g. [5, (5]). However, concerning the possible existence and stability of spatially periodic SWs in anharmonic lattices, much less has been known up to now. Although stable SW solutions of the form $A \cos (Q n) \cos (\omega t)$ generally exist in harmonic lattices as linear superpositions of counterpropagating waves with equal amplitudes $A$ and frequencies $\omega$ but opposite wave numbers $\pm Q$, there is a priori no guarantee that these SWs will remain stable in the presence of anharmonicity.

Here, we consider the general class of one-dimensional (1D) anharmonic lattices described by a discrete nonlinear Klein-Gordon (KG) or Schrödinger (DNLS) equation. We first propose a method to generate (numerically) exact time-periodic SW solutions to these equations. These SWs, whose spatial periodicity can be either commensurate $(Q / 2 \pi$ rational) or incommensurate $(Q / 2 \pi$ irrational) with the lattice period, are found as 'multibreather' solutions [5] from the so called 'anticontinuous' limit of zero inter-site coupling, and they reduce to the harmonic SWs for small amplitudes. Then, we investigate the stability properties of these SWs, and find as a most striking result that for infinite lattices, SWs with small but nonzero amplitude are unstable through an oscillatory instability for all $0<Q<\pi$ [7]. Thus, the SWs are unstable also for wave numbers where MI for propagating waves does not occur. It is important to note that the additional SW instabilities appear as a direct consequence of the lattice discreteness, and have not been found within continuum approximations [8]. Moreover, the dynamics resulting from the SW instabilities is fundamentally different from that of the MI, as the latter generally is non-oscillatory (characterized by purely imaginary eigenfrequencies of the linearized equations (4) below) while the former is oscillatory (corresponding to complex eigenfrequencies with nonzero real part) [9]. In this Letter, our main results will be stated and motivated briefly; full technical details will be given elsewhere [10].

We thus consider a 1D chain of classical anharmonic oscillators with a general on-site potential $V(u)$ and harmonic inter-site coupling $C_{K}>0$, yielding a discrete nonlinear KG equation for the particle displacements $u_{n}$,

$$
\ddot{u}_{n}+V^{\prime}\left(u_{n}\right)-C_{K}\left(u_{n+1}+u_{n-1}-2 u_{n}\right)=0 .
$$

Here, we shall be mainly concerned with the smallamplitude dynamics of KG chains with small inter-site coupling $C_{K}$, which can be well described by a DNLS approximation [3, 8, 10 (its range of validity is investigated in more details in [10]). For small oscillations, the on-site potential $V(u)$ can be expanded as $V(u)=u^{2} / 2+\alpha u^{3} / 3$ $+\beta u^{4} / 4+\ldots$, where the linear oscillator frequency is set to 1. Linearizing Eq. (11) yields propagating or SW solutions with wave number $Q$ and frequency $\omega_{0}(Q)$ given by the dispersion relation $\omega_{0}^{2}(Q)=1+4 C_{K} \sin ^{2} Q / 2$. For the nonlinear Eq. (11), we search for small-amplitude timeperiodic solutions as $u_{n}(t)=\sum_{p} a_{n}^{(p)} e^{i p \omega_{b} t}$, for which the dynamics will be almost harmonic with frequency $\omega_{b}$ close to $\omega_{0}(Q)$ for some $Q$. Then, $\omega_{b}^{2}-1 \equiv 2 \delta$ will be of order $C_{K}$, and allowing for a slow time dependence of the Fourier coefficients $a_{n}^{(p)}$ yields [10], at order $C_{K}$, a DNLS equation for the dominating coefficient $a_{n}^{(1)}$ describing the leading-order nonlinear effects. Defining $\lambda=$ $-5 \alpha^{2} / 3+3 \beta / 2$ (so that for soft [hard] potentials, $\lambda<0$ $[\lambda>0])$ and $\psi_{n}=\sqrt{|\lambda|} a_{n}^{(1)}$, the DNLS equation reads

$$
i \dot{\psi}_{n}=\delta \psi_{n}-\sigma\left|\psi_{n}\right|^{2} \psi_{n}+C\left(\psi_{n+1}+\psi_{n-1}-2 \psi_{n}\right),
$$

with $\sigma=\operatorname{sign}(\lambda)$ and $C=C_{K} / 2$. We will assume $\sigma=-1$ 
without loss of generality, since changing $\psi_{n} \rightarrow(-1)^{n} \psi_{n}^{*}$, $\delta \rightarrow 4 C-\delta$ is equivalent to changing the sign of $\sigma$.

For time-periodic solutions all $a_{n}^{(p)}$, and therefore $\psi_{n}$, will be time-independent and can be chosen real. Then, vanishing the left-hand side of Eq. (2) defines a nonlinear symplectic map $\mathcal{S}:\left(\psi_{n}, \psi_{n-1}\right) \rightarrow\left(\psi_{n+1}, \psi_{n}\right)$ in the $2 \mathrm{D}$ real plane, which, with the change of scale $\psi_{n} \rightarrow \sqrt{C} \psi_{n}$, depends only on the parameter $\delta^{\prime} \equiv \delta / C$. As is wellknown [11], this map exhibits a rich variety of orbits, including elliptic and hyperbolic fixpoints and periodic cycles, KAM tori, Aubry-Mather Cantor sets (Cantori) 12] and chaotic orbits. Searching for nonlinear SWs in continuation of the linear SWs $\psi_{n}=A \cos (Q n+\phi)$, with $\delta=4 C \sin ^{2} Q / 2 \equiv \delta_{0}(Q)$, only the periodic and quasiperiodic orbits which can be continued to zero amplitude by varying $\delta^{\prime}$ are of interest. For small amplitudes, these orbits must be located close to the elliptic fixpoint $F_{0}=(0,0)$, and $Q$ must be close to its linear rotation number $\theta\left(\delta^{\prime}\right)$ given by $\delta=\delta_{0}(\theta)$. As $\sigma<0$, the rotation number for orbits rotating around $F_{0}$ increases with increasing radius, and since the map $\mathcal{S}$ as well as its orbits at fixed rotation number depends continuously on $\delta^{\prime}, S W s$ with wave number $Q$ exist only for $\delta \leq \delta_{0}(Q)$.

When $Q=2 \pi r / s$ ( $r$ and $s$ irreducible integers), there are two families of commensurate $S W s$, each of them represented by one of the two $s$-periodic cycles (hereafter called ' $h$-cycle' resp. 'e-cycle') which bifurcate in pair from $F_{0}$ at $\delta=\delta_{0}(Q)$ 11 13. The h-cycle is hyperbolic for all $\delta^{\prime}$, while the e-cycle is elliptic for $\delta$ close to $\delta_{0}(Q)$ and hyperbolic with reflection below a critical value $\delta=\delta_{c}(Q)$. When $Q / 2 \pi$ is a generic irrational number, the trajectories representing incommensurate SWs emerge from $F_{0}$ as the KAM torus with rotation number $Q$. At the critical value $\delta=\delta_{c}(Q)$, the KAM torus breaks up in a transition by breaking of analyticity 12 and bifurcates into a Cantorus and its associated 'midgap' trajectory 14. Thus, for $\delta<\delta_{c}(Q)$ the Cantorus and the midgap trajectory define two families of non-analytic incommensurate $S W s$, which merge into the unique analytic $S W$ defined by the KAM torus for $\delta \geq \delta_{c}(Q)$.

To give a global representation of these SWs, we consider the large-amplitude limit $\delta \rightarrow-\infty$, or, equivalently, the anticontinuous limit $C=0$ for fixed $\delta<0$. From this limit, where stationary solutions to Eq. (2) can only take the values $\psi_{n}= \pm \sqrt{-\delta}$ and $\psi_{n}=0$, we can describe the SWs as multibreathers 13,5] identified by a coding sequence $\sigma_{n}^{\prime}$ defined as $\psi_{n}=\sqrt{-\delta} \sigma_{n}^{\prime}$. By continuing the SW map orbits to large negative $\delta^{\prime}$, we find their coding sequences as $\sigma_{n}^{\prime}=\chi_{0}(Q n+\phi)$, where $\chi_{0}(x)$ is the $2 \pi$-periodic odd function defined for $x \in[0, \pi]$ as

$$
\chi_{0}(x)=\left\{\begin{array}{ll}
1 & \text { for }(\pi-Q) / 2 \leq x \leq(\pi+Q) / 2 \\
0 & \text { elsewhere }
\end{array} .\right.
$$

For all phases $\phi \neq \phi_{m} \equiv \pm(\pi-Q) / 2-m Q$ ( $m$ integer), this describes SWs corresponding either to the hcycles (for rational $Q / 2 \pi$ ) or to the Cantori (for irrational
$Q / 2 \pi)$. We will call these SWs 'type $H$ ', and they have the property (of importance for the stability analysis below) that their coding sequences do not contain any consecutive +1 or -1 . For the particular phases $\phi=\phi_{m}$, $x=Q m+\phi$ is at a discontinuity point of $\chi_{0}(x)$, so that $\sigma_{n}^{\prime}$ has two consecutive +1 (or -1$)$. These SWs, called type $E$, correspond to e-cycles or to midgap trajectories.

The representation (3) is useful also for numerically calculating directly the exact SWs of the original KG equation (11), using standard methods (e.g. Newton schemes) for continuation of multibreathers from the anticontinuous limit. Continuing these solutions versus $\omega_{b}$ to zero amplitude, we typically find (Fig. 11) a transition by breaking of analyticity of the hull function $\chi(x)$, defined as $u_{n}(0)=\chi(Q n+\phi)$, for incommensurate SWs in the KG chains as well as in DNLS chains.
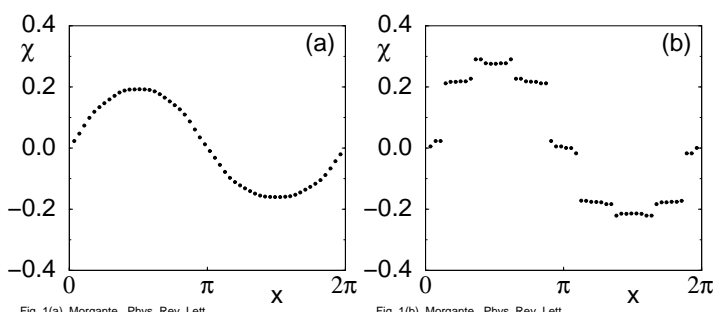

FIG. 1. Hull functions $\chi(x)$ for SW SW in a KG chain (何) with $V(u)=\frac{1}{2}\left(e^{-u}-1\right)^{2}, C_{K}=0.05, Q / 2 \pi=21 / 55 \simeq(3-\sqrt{5}) / 2$ $\equiv \sigma_{G}$ and (a) $\omega_{b}=1.072$ resp. (b) $\omega_{b}=1.060$. The breaking of analyticity occurs at $\omega_{b} \simeq 1.068$ for $Q / 2 \pi=\sigma_{G}$.

We now turn to the investigation of the dynamical stability properties of these SWs. As before, we consider small-amplitude solutions to the KG equation (11) with small $C_{K}$, so that the DNLS approximation (2) is well justified. With the substitution $\psi_{n} \rightarrow \psi_{n}+\epsilon_{n}(t)$, where $\psi_{n}$ is real and time-independent and $\epsilon_{n}$ is small, the linearization of Eq. (2) yields a standard Hill equation for $\epsilon_{n}$. Its eigenmodes can be found by setting $\epsilon_{n}(t)=\frac{1}{2}\left(u_{n}+v_{n}\right) e^{i \omega_{l} t}+\frac{1}{2}\left(u_{n}^{*}-v_{n}^{*}\right) e^{-i \omega_{l} t}$, where the eigenfrequencies $\omega_{l}$ are given by the eigenvalue problem:

$$
\begin{gathered}
\mathcal{L}_{0} v_{n} \equiv\left(2 C-\delta-\psi_{n}^{2}\right) v_{n}-C\left(v_{n+1}+v_{n-1}\right)=\omega_{l} u_{n} \\
\mathcal{L}_{1} u_{n} \equiv\left(2 C-\delta-3 \psi_{n}^{2}\right) u_{n}-C\left(u_{n+1}+u_{n-1}\right)=\omega_{l} v_{n}
\end{gathered}
$$

The SW $\psi_{n}$ is linearly stable if and only if all eigenfrequencies $\omega_{l}$ are real.

At the anticontinuous limit $(\delta<0, C=0)$, the eigenvalues of (4) will be located either at $\omega_{l}=0$ (corresponding to sites with codes $\left|\sigma_{n}^{\prime}\right|=1$ ) or at $\omega_{l}= \pm \delta$ (corresponding to $\sigma_{n}^{\prime}=0$ ). For commensurate SWs $(Q=2 \pi r / s)$ the lattice periodicity of $\psi_{n}^{2}$ in (A) implies that the degenerate eigenvalues will form bands as $C$ is increased from zero (see Fig. 2). There will be $s-2 r$ pairs of bands originating from $\omega_{l}= \pm \delta$, and $r$ pairs of bands originating from $\omega_{l}=0$ (which always remains in the spectrum). For incommensurate SWs, the band structure will be Cantor-like. In all cases, bands originating 
from $\pm \delta$ can be shown to move initially only along the real axis when $C$ is increased from zero, while the behaviour of the bands originating from 0 will be different for $\mathrm{E}$ and $\mathrm{H}$ type SWs. As the coding sequences for $\mathrm{E}$ type SWs contain pairs of consecutive +1 (or -1 ) and the anharmonicity is soft, it follows from a general result [5] that some eigenvalues always move out on the imaginary axis as soon as $C \neq 0$, making these SWs dynamically unstable for $\delta<0$ and small $C$. Moreover, we find [10] that this instability persists for all $\delta<\delta_{0}(Q)$, so that SWs of type $\mathrm{E}$ are unstable for any nonvanishing amplitude. By contrary, for $\mathrm{H}$ type SWs the theory of effective action [5] can be used to show that all eigenvalues initially will move along the real axis, and thus the stability of these $S W$ s is preserved for $C$ not too large (Fig. 目 (a)).

Increasing $\delta$ (for fixed $C$ ) the bands broaden, and the main gap separating the two classes of bands originating from 0 resp. $\pm \delta$ shrinks. At some value $\delta=\delta_{K}(Q)$ it vanishes, and, as shown in Fig. 2(b), eigenvalues from the two classes of bands will collide and move out in the complex plane (since they have opposite Krein signature [5]). Thus, oscillatory instabilities appear for the $S W$. These instabilities have also been confirmed by direct numerical Floquet analysis of the KG Eq. (11) for small $C_{K}$ [10].
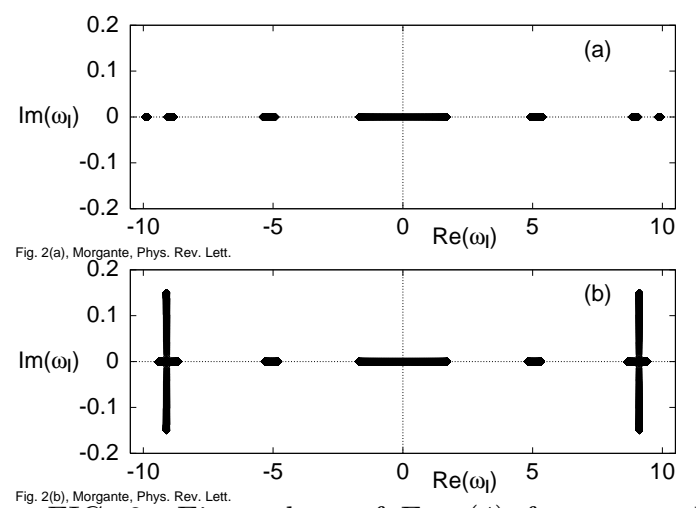

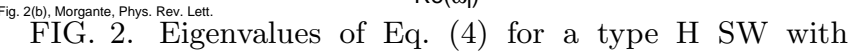
$Q=3 \pi / 4$ and (a) $\delta^{\prime}=-8.0$ resp. (b) $\delta^{\prime}=-7.5(C=1)$.

For $\delta>\delta_{K}(Q)$ bands continue to overlap, generating new instabilities. In many cases, the SW remains unstable until it vanishes at $\delta=\delta_{0}(Q)$, although for some $Q$ with small $s$ the stability is temporarily regained for some interval of $\delta$ where no bands overlap. But for all commensurate SWs, there is a final interval $\delta_{1}(Q) \leq \delta<\delta_{0}(Q)$ where bands with opposite Krein signature overlap, and as a consequence all commensurate $S W$ s are unstable for small but nonzero amplitude. This can be proven [10] by considering the linear limit $\left(\delta=\delta_{0}(Q), \psi_{n}^{2}=0\right)$, where the spectrum of (1) is given by $\omega_{l}= \pm 2 C\left|\nu_{Q}(q)\right|$ with $\nu_{Q}(q)=\cos Q-\cos q$. In this limit, eigenvalues with opposite Krein signature (given by $\operatorname{sign}\left(\nu_{Q}(q)\right)$ [10]) will overlap in an interval around $\omega_{l}=0$. Since for commensurate SWs a finite number of bands with nonzero widths appear for $\delta<\delta_{0}(Q)$ when $\psi_{n}^{2} \neq 0$, a continuity argument [10] yields that bands with opposite Krein signature will continue to overlap close to $\omega_{l}=0$ and cause instabilities until $\delta$ reaches some value $\delta_{1}(Q)<\delta_{0}(Q)$. (In the linear limit, the SW is stable in spite of the band overlap, since the eigenmodes are uncoupled.)

By contrast, for small-amplitude (analytic) incommensurate SWs the Cantor-like nature of the spectrum of (4) (with 'bands' of zero width) prevents the use of a similar argument for proving instability. However, the analysis outlined below shows [10] that also these SWs are unstable, but the instabilities may be of higher order and thus more difficult to detect numerically or experimentally.

The instability of analytic SWs is proven using the method of 'band analysis' 5 . Its basic idea is to embed the eigenvalue problem (4) for a non-Hermitian operator into a wider eigenvalue problem for a Hermitian operator, which we choose as (with $\omega$ a real parameter)

$$
\left(\begin{array}{cc}
\mathcal{L}_{1} & -\omega \\
-\omega & \mathcal{L}_{0}
\end{array}\right)\left(\begin{array}{l}
\left\{u_{n}\right\} \\
\left\{v_{n}\right\}
\end{array}\right)=E\left(\begin{array}{c}
\left\{u_{n}\right\} \\
\left\{v_{n}\right\}
\end{array}\right)
$$

Then, each eigenvalue $E_{\nu}$ is a smooth, real function ('band') of $\omega$. The real eigenfrequencies $\omega_{l}$ of (4) are given by the intersections of these bands with $E=0$, and an instability occurs whenever a band loses a pair of intersections with $E=0$, i.e., when a 'gap' opens around $E=0$.

In the linear limit $\left(\psi_{n} \rightarrow 0, \delta \rightarrow \delta_{0}(Q)\right)$ the bands can be labelled by a wave number $q$ and describe straight lines $E_{ \pm}(q ; \omega)=2 C(\cos Q-\cos q) \pm \omega$. Thus, for each $q$ there are two bands (doubly degenerate since $E_{ \pm}(q ; \omega)=$ $\left.E_{ \pm}(-q ; \omega)\right)$, and bands with different $q$ intersect when $E_{-}\left(q_{-} ; \omega\right)=E_{+}\left(q_{+} ; \omega\right)$. When $\psi_{n}$ describes an analytic SW with small amplitude $A, \psi_{n}^{2}$ can be treated as a perturbation and expanded as $\psi_{n}^{2}=\sum_{p} f_{p} e^{i p(2 n Q+\phi)}$, where the coefficients $f_{p}$ are of order $A^{2|p|}$ for $|p| \geq 1$, and thus exponentially decaying for $p \rightarrow \infty$. This introduces a coupling between eigenvectors of (5) with wave numbers $q$ and $q+2 p Q$ with coupling strength proportional to $f_{p}$, and gaps of width $\Delta E \propto 2\left|f_{p}\right|$ will open at each intersection where $E_{-}(q ; \omega)=E_{+}(q+2 p Q ; \omega)$. If these gaps surround the axis $E=0$, instabilities occur for the SW.

For fixed $p$ these intersections describe an ellipse in the $(\omega, E)$-plane given by

$$
\left(\frac{\omega_{p}}{2 C \sin p Q}\right)^{2}+\left(\frac{E_{p}-2 C \cos Q}{2 C \cos p Q}\right)^{2}=1 .
$$

Numerical calculations of the set of bands $E(\omega)$ confirm the existence of these ellipses of gaps (see Fig. 3). The ellipse with $p=1$ corresponds to the largest gaps (and thus the strongest instabilities if the gaps surround $E=0$ ) and is for $\delta=\delta_{0}(Q)$ tangent to $E=0$ at $\omega=0$, lying below $E=0$ for $\pi / 2<Q<\pi$ and above for $0<Q<\pi / 2$. Considering also the shift of the bands caused by the static term $f_{0}$, we find 10 that when $0<Q<\pi / 2$ gaps will open around $E=0$ in the lower part of the ellipse, 
and thus these waves become immediately unstable to first order (as for commensurate SWs with $Q \neq \pi$ [10]).

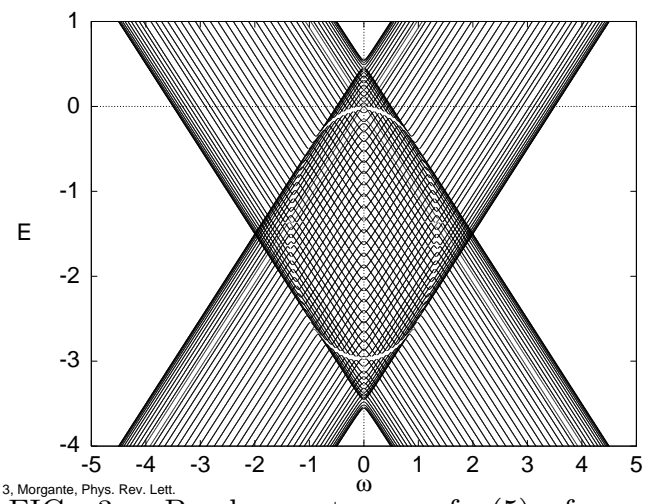

FIG. 3. B. Band spectrum of (B) for a $\mathrm{SW}$ with $Q / 2 \pi=34 / 89 \simeq \sigma_{G}(Q>\pi / 2)$ and $\delta^{\prime}=3.4(C=1)$. The visible gap openings occur along the ellipse (6) with $p=1$.

By contrast, for irrational $Q$ with $\pi / 2<Q<\pi$ all first-order gaps open strictly below $E=0$ (Fig. [3), so that no first order instabilities develop for these SWs. However, as is seen from (6), the ellipse corresponding to the $p$ th order intersections will intersect $E=0$ if $|\cos p Q|$ $>|\cos Q|$, and therefore there is always a $p$ such that the $S W$ is unstable to order $p$ for arbitrarily small amplitude. But as the width of the gaps and the maximum instability growth rates are proportional to $\left|f_{p}\right|$, the instabilities become very weak when the smallest $p$ yielding an instability becomes large (for $Q$ close to $\pi$ ). We might therefore view these analytic SWs as 'quasi-stable'. A typical example of the dynamics resulting from this instability in

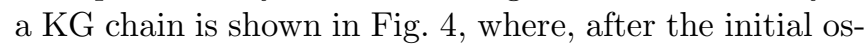
cillatory dynamics and an intermediate regime of mainly translational motion, an apparently chaotic final state appears. Further details concerning the long-time dynamics of the unstable SWs will be published elsewhere.

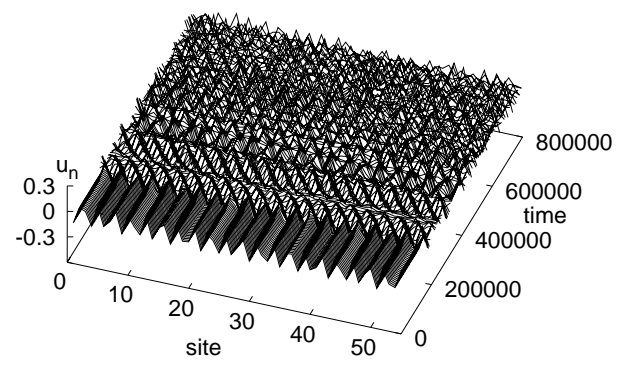

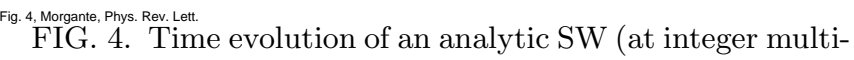
ples of the original period), perturbed only by numerical truncation errors, in a KG chain (1) with $C_{K}=0.03, \omega_{b}=1.044$, and other parameters as in Fig. 1.

In conclusion, we have shown that for a general class of anharmonic lattices, different types of SWs with spatial period commensurate or incommensurate with the lat- tice exist, but are generically unstable through oscillatory instabilities for small amplitudes. Thus, in discrete systems, the nonlinearity-induced coupling between counterpropagating waves leads to wave break-down for arbitrarily weak anharmonicity even if the individual propagating waves are modulationally stable. The general nature of this result suggests that these instabilities should be observable in macroscopic as well as microscopic contexts. In particular, as was recently experimentally verified [15], nonlinear optical waveguide arrays provide a direct application of the DNLS equation, and thus these systems are also good candidates for detection of SW instabilities.

M.J. acknowledges a EC Marie Curie fellowship.

[1] E. Infeld and G. Rowlands, Nonlinear Waves, Solitons, and Chaos (Cambridge University Press, 1990).

[2] See, e.g., N. N. Akhmediev, V. M. Eleonskii, and N. E. Kulagin, Theor. Math. Phys. 72, 809 (1987).

[3] Yu. S. Kivshar and M. Peyrard, Phys. Rev. A 46, 3198 (1992); I. Daumont, T. Dauxois, and M. Peyrard, Nonlinearity 10, 617 (1997).

[4] T. Cretegny and S. Aubry, Phys. Rev. B 55, R11929 (1997).

[5] S. Aubry, Physica D 103, 201 (1997).

[6] S. Flach and C. R. Willis, Phys. Rep. 295, 181 (1998).

[7] As will be discussed in [10], these instabilities are very sensitive to finite size effects, similarly as found e.g. by J. L. Marín and S. Aubry, Physica D 119, 163 (1998).

[8] Yu. S. Kivshar, M. Haelterman, and A. P. Sheppard, Phys. Rev. E 50, 3161 (1994).

[9] A similar instability mechanism has been found in the DNLS chain for 'discrete dark solitons' (which can be viewed as a limit case of SWs [10]), see M. Johansson and Yu. S. Kivshar, Phys. Rev. Lett. 82, 85 (1999).

[10] A. M. Morgante et al., in preparation.

[11] See e.g. Yi Wan and C. M. Soukoulis, Phys. Rev. A 41, 800 (1990); D. Hennig and G. P. Tsironis, Phys. Rep. 307, 333 (1999), and references therein.

[12] S. Aubry, in Solitons and Condensed Matter Physics, ed. A. R. Bishop and T. Schneider (Springer, 1978), p. 264 (1978); S. Aubry, in Structure et Instabilité, ed. C. Godrèche, Edition de Physique, p. 73 (1986) (in French).

[13] S. Aubry, in Twist Mappings and their Applications, eds. R. McGehee and K. R. Meyer (Springer, 1992), p. 7; S. Aubry and G.Abramovici, Physica 43D, 199 (1990).

[14] The midgap trajectory consists of isolated, nonrecurrent points in the gaps of the Cantorus with rotation angle $Q$, to which it is homoclinic. See J. Mather, Publ. Math. IHES 63, 153 (1986); A. Katok and B. Hasselblatt, Introduction to the Modern Theory of Dynamical Systems (Cambridge University Press, 1995), p. 441.

[15] D. N. Christodoulides and R. I. Joseph, Opt. Lett. 13, 794 (1988); H. S. Eisenberg et al., Phys. Rev. Lett. 81, 3383 (1998); R. Morandotti et al., ibid. 83, 2726 (1999). 